\title{
Impact des décès par surdose de drogue sur l'espérance de vie à la naissance en Colombie-Britannique
}

\author{
Xibiao Ye, Ph. D. (1); Jenny Sutherland, M. Sc. (1); Bonnie Henry, M.D. (1); Mark Tyndall, M.D. (2); \\ Perry Robert William Kendall, M.D. (1)
}

Diffuser cet article sur Twitter

\section{Résumé}

Nous avons quantifié la contribution des principales causes de décès et de surdose à l'évolution de l'espérance de vie à la naissance et aux disparités en matière de sexe et de situation socioéconomique en Colombie-Britannique. Entre 2014 et 2016, l'espérance de vie à la naissance a diminué de 0,38 an et les décès par surdose (principalement liés aux opioïdes) ont contribué à ce recul pour 0,12 an. L'analyse a également montré que le taux plus élevé de mortalité par surdose constaté chez les hommes et les membres des catégories socioéconomiques plus défavorisées a contribué à une diminution différentielle dans ces deux groupes de l'espérance de vie à la naissance.

Mots-clés : Décès par surdose d'opioüdes, espérance de vie à la naissance, inégalité

\section{Introduction}

Le nombre de décès par surdose de drogue illicite a considérablement augmenté en Colombie-Britannique depuis 2014, passant de 369 à 1208 décès (compte tenu des cas suspectés) au 31 octobre $2017^{1}$. Le fentanyl et ses analogues, en combinaison avec d'autres drogues, sont à l'origine de la majeure partie de ces décès ${ }^{2}$. En réaction à l'aggravation de cette crise des surdoses, une urgence en santé publique a été déclarée le 14 avril 2016 en ColombieBritannique $^{3}$.

La contribution des décès par surdose aux variations de l'espérance de vie a rarement été quantifiée. Entre 2000 et 2014, les intoxications accidentelles (le plus souvent par surdose de drogue ou d'alcool) ont fait perdre 0,338 an d'espérance de vie à la naissance au sein de la population blanche d'origine non hispanique aux États-Unis. C'est là l'incidence négative la plus marquante par cause de décès ${ }^{4}$. Les décès par surdose d’opioïdes ont représenté plus spécifiquement une perte de 0,21 an d'espérance de vie à la naissance entre 2000 et 2015 à l'échelle de la population américaine $^{5}$. Dans cet article, nous avons voulu appliquer cette analyse au contexte de la Colombie-Britannique et l'approfondir en chiffrant la contribution qu'apportent les décès par surdose d'opioïdes ou d'autres drogues aux disparités en matière d'espérance de vie selon le sexe et la situation socioéconomique (SSE).

\section{Méthodologie}

Nous avons obtenu les données sur les décès relevés par le bureau de l'état-civil de Colombie-Britannique (BC Vital Statistics Agency) pour 2001-2016. Nous avons utilisé la Classification statistique internationale des maladies, traumatismes et causes de décès, $10^{\mathrm{e}}$ révision (CIM-10) pour en classer les causes. Nous avons dénombré les décès liés aux opioïdes (T40.0, T40.1, T40.2, T40.3, T40.4 et T40.6), à la cocaïne (T40.5) et à d'autres drogues (T40.7, T40.8 et T40.9). Les décès causés

\section{Points saillants}

- L'espérance de vie à la naissance en Colombie-Britannique a diminué de 0,38 an entre 2014 et 2016, et les surdoses mortelles de drogue (la majorité mettant en cause des opioïdes) expliquent $32 \%$ de cette baisse.

- En 2016, l'espérance de vie à la naissance des hommes était de 4,59 ans inférieure à celle des femmes, et la mortalité par surdose contribuait à cet écart pour $9 \%$.

- En 2016, l'espérance de vie à la naissance dans les collectivités ayant l'indice de défavorisation le plus élevé (quintile 5 ou situation socioéconomique la plus basse) était inférieure de 5,58 ans comparativement aux membres des collectivités ayant l'indice de défavorisation le plus faible (quintile 1 ou situation socioéconomique la plus élevée), et la mortalité par surdose contribuait à cet écart pour $7 \%$.

par des blessures involontaires (X40-X44) ou relevant d'une intention indéterminée (Y10-Y14) ont été inclus dans l'analyse. Nous avons calculé les taux de mortalité en nous reportant à la population assurée de la province et nous avons pris la population de 2001 comme référence pour normaliser les taux.

Nous avons appliqué la méthode de Chiang $^{6}$ pour la construction des tables de

Rattachement des auteurs :

1. Office of the Provincial Health Officer, ministère de la Santé de la Colombie-Britannique, Victoria (Colombie-Britannique), Canada

2. BC Centre for Disease Control, Vancouver (Colombie-Britannique), Canada

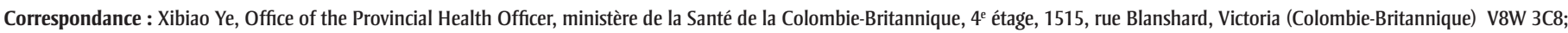
tél. : 250-952-2026; courriel : xibiao.ye@gov.bc.ca 
survie par période et calculé les écarts d'espérance de vie à la naissance entre 2001 et 2016 et entre 2014 et 2016. Nous avons examiné les disparités d'espérance de vie à la naissance selon le sexe et l'indice de défavorisation. Cet indice, qui livre une mesure régionale de la SSE incluant une dimension matérielle (variable composite du revenu du ménage, du chômage et de l'achèvement des études secondaires) et une dimension sociale (variable composite de l'état matrimonial, du fait de vivre seul et de la stabilité résidentielle), a été construit à l'aide des données du recensement du Canada de 2011 en utilisant la méthode employée par Pampalon et ses collaborateurs ${ }^{7}$. Une valeur faible de l'indice correspond à une meilleure situation socioéconomique (c'est-à-dire à moins de défavorisation). Nous avons réparti les écarts en fonction de l'âge et des principales causes de décès, dont les surdoses, au moyen de la méthode de décomposition d'Arriaga $^{8}$. Nous avons utilisé SAS version 9.3 (SAS Institute Inc., Cary, Caroline du Nord, États-Unis) pour nos analyses.

\section{Résultats}

En Colombie-Britannique, l'espérance de vie à la naissance a augmenté entre 2001 et 2014, passant de 80,27 ans (intervalle de confiance [IC] à $95 \%: 80,12$ à 80,42 ) à 83,02 ans (IC à $95 \%$ : 82,88 à 83,16). Entre 2014 et 2016, elle a diminué de 0,38 an pour atteindre 82,64 ans (IC à $95 \%$ : 82,50 à 82,77 ) (tableau 1). La réduction des taux de mortalité par cancer, cardiopathie, maladie cérébrovasculaire et accident a contribué en majeure partie à cette

TABLEAU 1

Contributions des principales causes de décès et de surdose de drogue à l'évolution de l'espérance de vie à la naissance en Colombie-Britannique

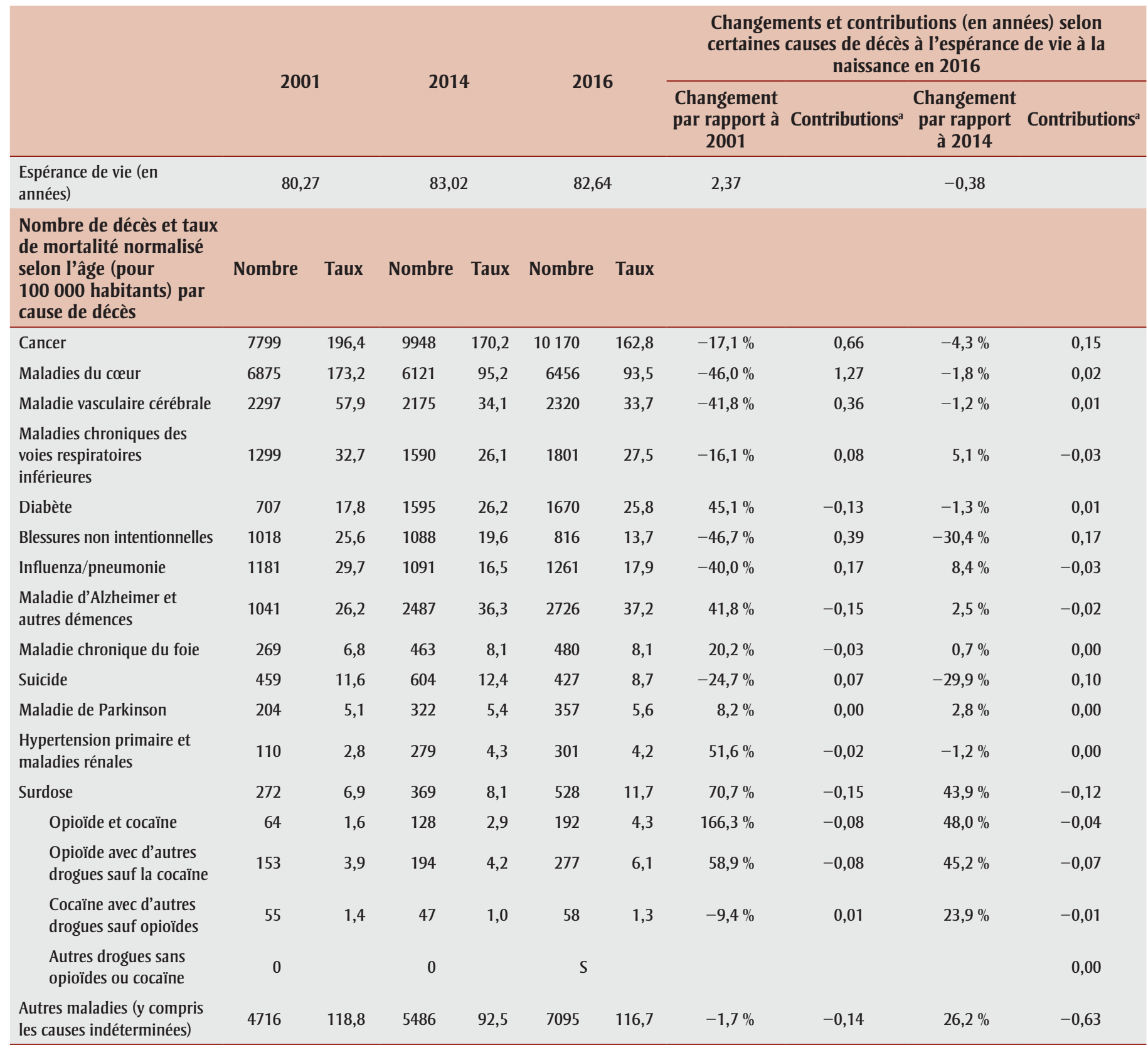

Abréviation : $\mathrm{S}$, valeur supprimée car le nombre de décès est de moins de 5.

${ }^{a}$ La valeur de la contribution est négative quand le taux de mortalité imputable à une cause a augmenté au fil des ans en diminuant donc l'espérance de vie à la naissance. 
progression de 2,37 ans de l'espérance de vie à la naissance entre 2001 et 2016. Il reste que les décès liés à la drogue ont fait reculer cette espérance de 0,15 an au cours de l'ensemble de la période. Les décès liés spécifiquement aux opioïdes constituaient près de $80 \%$ de l'ensemble des décès par surdose en 2001, et ce pourcentage est passé à $90 \%$ en 2016. L'augmentation du nombre de décès attribuables aux opioïdes a entraîné une perte de 0,16 an de l'espérance de vie à la naissance entre 2001 et 2016. Les décès par surdose lui ont fait perdre 0,12 an entre 2014 et 2016, soit $32 \%$ du recul sur cette période.

En 2001, l'espérance de vie à la naissance des hommes était de 5,01 ans inférieure à celle des femmes (tableau 2). Le taux plus élevé de mortalité masculine par surdose expliquait 0,20 an de l'écart, mais dans la majorité des cas la cause du décès était attribuable aux cancers, aux cardiopathies ou aux blessures (accidents et suicides). Alors que la disparité d'espérance de vie à la naissance entre les sexes a baissé pour atteindre 4,59 ans en 2016, la contribution des décès par surdose a dans le même temps doublé pour atteindre 0,42 an $(9 \%$ de l'écart). Les taux de mortalité par surdose se sont révélés en relation inverse avec l'indice de défavorisation matérielle et sociale. En 2011, l'espérance de vie à la naissance pour la population présentant le niveau global de défavorisation le plus élevé (dernier quintile ou SSE la plus basse) était de 5,50 ans inférieure à celle de la population ayant le niveau global de défavorisation le plus faible (premier quintile ou SSE la plus élevée). De cette valeur, 0,31 an était attribuable aux décès par surdose. En 2016, la contribution des décès par surdose est passée à 0,39 an (soit $7 \%$ de l'écart global). Aux deux dates, les disparités liées à la défavorisation sociale l'emportaient sur les disparités liées aux disparités matérielles.

\section{Analyse}

Nos analyses nous ont permis de mesurer une augmentation de 2,37 ans de l'espérance de vie à la naissance entre 2001 et 2016, mais une diminution de 0,38 an entre 2014 et 2016 (dont 0,12 an attribuable aux décès par surdose). Alors que la disparité d'espérance de vie à la naissance selon le sexe a légèrement diminué entre 2001 et 2016, la contribution des décès par surdose à cette disparité a doublé. Entre 2011 et 2016, la disparité d'espérance de vie à la naissance en fonction du niveau de défavorisation (entre les quintiles 1 et 5 ) est demeurée relativement stable, mais la contribution des décès par surdose à cette disparité s'est élevée.

Entre 2000 à 2015, les surdoses ont fait perdre 0,28 an d'espérance de vie à la naissance aux États-Unis. Sur ce total, 0,21 an était attribuable aux décès par surdose d'opioïdes ${ }^{5}$. Dans cette analyse, nous avons démontré que les décès par surdose, en particulier d'opioïdes, ont fait reculer considérablement l'espérance de vie à la naissance en Colombie-Britannique. Toutefois, cette contribution a été plus faible que pour l'ensemble des États-Unis du fait de moindres taux comparatifs de mortalité (ainsi, le taux de mortalité par surdose d'opioïdes pour les deux sexes était de 16,3 pour 100000 Américains en $2015^{5}$ et de 11,9 pour 100000 BritannoColombiens en 2016). L'espérance de vie à la naissance s'est améliorée ces dernières décennies aux États-Unis pour culminer à 78,9 ans en 2014, mais a ensuite fléchi légèrement pour atteindre 78,8 ans en 2015 et 78,6 en 2016. Cette baisse est largement attribuable à l'augmentation du nombre de décès chez les plus jeunes et de décès par blessures involontaires, dont par surdose de drogue ${ }^{4,9}$. De manière similaire, nous avons constaté une diminution en Colombie-Britannique depuis 2014 et celle-ci est attribuable en partie à une progression du nombre de décès par surdose, en particulier chez les hommes. D'autres provinces ont également vu progresser le nombre de décès par surdose $\mathrm{e}^{10} \mathrm{à} 12$, mais on ne sait pas au juste quel en sera l'effet sur l'espérance de vie à l'échelle nationale.

Il a été fait état de disparités d'espérance de vie à la naissance selon le sexe et selon la situation socioéconomique à différents niveaux géographiques ${ }^{13-16}$. Ces études ont fait clairement ressortir des différences, mais sans que l'on sache véritablement quelle contribution apportent respectivement les causes de décès et les facteurs de risque associés au sexe et à la SSE. Dans cette analyse, nous avons montré que les décès par surdose expliquaient en 2016 à eux seuls environ $9 \%$ des pertes relatives en matière d'espérance de vie à la naissance entre les hommes et les femmes. Cette contribution a doublé au cours des 15 dernières années en raison de l'augmentation appréciable du nombre de décès par surdose chez les hommes. Le taux de mortalité par surdose était trois

TABLEAU 2

Contribution des surdoses de drogue aux disparités d'espérance de vie à la naissance (en nombre d'années) en fonction du sexe et de l'indice de défavorisation, Colombie-Britannique

\begin{tabular}{|c|c|c|c|c|}
\hline \multirow[b]{2}{*}{ Facteur } & \multicolumn{2}{|c|}{2001 ou $2011^{a}$} & \multicolumn{2}{|c|}{2016} \\
\hline & $\begin{array}{c}\text { Différence d'espérance } \\
\text { de vie }\end{array}$ & Contribution des surdoses & $\begin{array}{c}\text { Différence d'espérance } \\
\text { de vie }\end{array}$ & Contribution des surdoses \\
\hline $\begin{array}{l}\text { Sexe (hommes par rapport aux } \\
\text { femmes) }^{b}\end{array}$ & $-5,01$ & $-0,20$ & $-4,59$ & $-0,42$ \\
\hline \multicolumn{5}{|l|}{$\begin{array}{l}\text { Indice de défavorisation (quintile } 5 \\
\text { par rapport au quintile 1) }{ }^{c}\end{array}$} \\
\hline Défavorisation matérielle & $-1,65$ & $-0,16$ & $-1,88$ & $-0,16$ \\
\hline Défavorisation sociale & $-5,62$ & $-0,26$ & $-5,43$ & $-0,33$ \\
\hline Défavorisation totale & $-5,50$ & $-0,31$ & $-5,58$ & $-0,39$ \\
\hline
\end{tabular}

${ }^{a}$ Années 2001 pour l'analyse selon le sexe et 2011 pour l'analyse par l'indice de défavorisation.

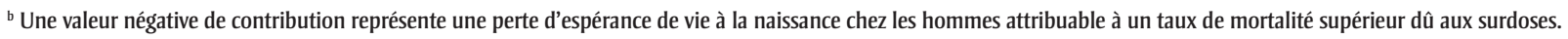

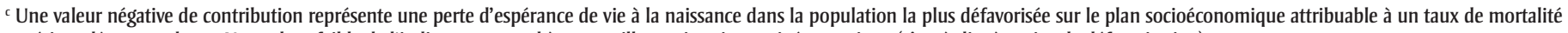
supérieur dû aux surdoses. Une valeur faible de l'indice correspond à une meilleure situation socioéconomique (c'est-à-dire à moins de défavorisation). 
fois plus élevé au sein des catégories socioéconomiques les plus défavorisées par rapport aux catégories les plus favorisées (données non présentées), représentant $7 \%$ de la perte d'espérance de vie à la naissance. Ces résultats illustrent l'effet marquant qu'ont eu les décès par surdose sur l'ensemble de la population de la Colombie-Britannique, et en particulier les différences de cet effet négatif dans le cas des hommes et des plus défavorisés sur le plan socioéconomique dans la province. Cela devrait affermir notre volonté de nous attaquer à cette cause de décès largement évitable.

La contribution des décès par surdose pourrait avoir été sous-estimée, car seuls les cas confirmés sont pris en compte et les coroners peuvent prendre jusqu'à deux ans et même plus pour établir la cause de décès. En 2016, le service des coroners de la Colombie-Britannique a signalé 985 décès par surdose de drogue ${ }^{1}$, mais en utilisant les données de l'état civil, nous en avons recensé 528 et nous avons dénombré plus de 1200 cas dont la cause de décès était indéterminée. Une proportion appréciable de ces cas indéterminés sera probablement considérée comme liée aux opioïdes, ce qui fera monter la contribution des décès par surdose d'opioïdes (probablement de plus de $50 \%$ ). Une étude récente indique en effet que, dans une proportion de $30 \%$, les décès imputables à la drogue dans le registre de toxicologie médicolégale de la Suède ne sont pas enregistrés dans la base des données de l'état civil de ce pays, d'où une sous-déclaration d'environ $20 \%$ de la mortalité liée aux drogues $^{17}$. En tenant compte d'autres sources d'information comme ce registre pour identifier des décès par drogue supplémentaires, on pourra affiner cette estimation.

\section{Conclusion}

L'espérance de vie à la naissance chez les habitants de la Colombie-Britannique a augmenté de trois ans entre 2001 et 2014, puis a diminué de 0,38 an de 2014 à 2016. La crise des surdoses d'opioïdes a grandement contribué à cette perte. La hausse du taux de mortalité liée aux surdoses d'opioïdes a également contribué à une diminution de l'espérance de vie chez les hommes comparativement aux femmes, et chez les membres des collectivités ayant l'indice de défavorisation le plus élevé comparativement aux membres des collectivités ayant l'indice de défavorisation le plus faible.

\section{Remerciements}

Les auteurs remercient M. Kim Reimer pour ses commentaires sur le manuscrit.

\section{Conflits d'intérêts}

Les auteurs n'ont aucun conflit d'intérêts à déclarer.

\section{Contributions des auteurs et avis}

XY a conceptualisé la méthodologie de l'étude et rédigé l'ébauche du manuscrit. JS a dirigé l'analyse des données. PK, BH et MT ont contribué à la conception de l'étude, à l'analyse et l'interprétation des données, et à la rédaction et la révision du manuscrit. Tous les auteurs ont lu et approuvé la version finale du manuscrit.

Le contenu de l'article et les points de vue qui y sont exprimés n'engagent que les auteurs et ne sont pas nécessairement représentatifs de ceux du gouvernement du Canada.

\section{Références}

1. Bureau du coroner en chef, BC Ministry of Public Safety and Solicitor General. Illicit Drug Overdose Deaths in BC. 2017. En ligne à : https:// www2.gov.bc.ca/assets/gov/public -safety-and-emergency-services /death-investigation/statistical/illicit -drug.pdf

2. Bureau du coroner en chef, BC Ministry of Public Safety and Solicitor General. Fentanyl-Detected Illicit Drug Overdose Deaths. 2017. En ligne à : https://www2.gov.bc.ca/assets/gov /public-safety-and-emergency-services /death-investigation/statistical/fentanyl -detected-overdose.pdf

3. BC Gov News. Provincial health officer declares public health emergency [Internet]. 2016 [consulté le 29 décembre 2017]. En ligne à : https://news.gov .bc.ca/releases/2016HLTH0026-000568

4. Kochanek KD, Murphy SL, Xu J, Arias E. Mortality in the United States, 2016 Key Findings Data from the National Vital Statistics System. NCHS Data Brief. 2016:293:1-8. En ligne à : https://www.cdc.gov/nchs /data/databriefs/db293.pdf
5. Dowell D, Arias E, Kochanek K, Anderson R, Guy GP, Losby JL et collab. Contribution of opioid-involved poisoning to the change in life expectancy in the United States, 2000-2015. JAMA. 2017;318(11):1065-1067. doi: 10.1001/jama.2017.9308.

6. Chiang C.L. On constructing current life tables. J Am Stat Assoc. 1972; 67(339):538-541.

7. Pampalon R, Hamel D, Gamache P, Philibert MD, Raymond G, Simpson A. Un indice régional de défavorisation matérielle et sociale pour la santé publique au Québec et au Canada. Revue canadienne de santé publique. 2012;103(8):17-22. En ligne à : http:// journal.cpha.ca/index.php/cjph/article /view/3156/2711

8. Arriaga EE. Measuring and explaining the change in life expectancies. Demography. 1984;21(1):83-96.

9. $\mathrm{Xu} \mathrm{J}$, Murphy SL, Kochanek KD, Arias E. Mortality in the United States, 2015 Key Findings Data from the National Vital Statistics System, Mortality. NCHS Data Brief. 2016; 267:3-8. En ligne à : https://www.cdc .gov/nchs/data/databriefs/db267.pdf

10. Santé publique Ontario. Morbidité et mortalité liées aux opioïdes en Ontario [Internet]. [consulté le 7 janvier 2018]. En ligne à : https://www .publichealthontario.ca/fr/dataand analytics/pages/opioid.aspx

11. Santé Alberta, Analytics and Performance Reporting Branch. Alberta Health Opioids and Substances of Misuse Alberta Report, 2017 Q3. Edmonton (Alb.) : Gouvernment of Alberta; 2017. En ligne à : https://open.alberta.ca /dataset/1cfed7da-2690-42e7-97e9 -da175d36f3d5/resource/e69ffcb0 - 6 d $34-4$ e 2 b-bf3 $5-7$ d 13 ec 35 de 59 /download/Opioids-Substances -Misuse-Report-2017-Q3.pdf

12. Agence de la santé publique du Canada (ASPC). Rapport national : décès apparemment liés à la consommation d'opioïdes (de janvier 2016 à mars 2017) [Internet]. Ottawa (Ont.) : ASPC; 2017 [consulté le 7 janvier 2018]. En ligne à : https://www .canada.ca/fr/sante-canada/services /toxiconomie/abus-medicaments -ordonnance/opioides/deces-lies -opioides.html 
13. Wang H, Naghavi M, Allen C, Barber RM, Bhutta ZA, Carter A et collab. Global, regional, and national life expectancy, all-cause mortality, and cause-specific mortality for 249 causes of death, 1980-2015: a systematic analysis for the Global Burden of Disease Study 2015. Lancet. 2016; 388(10053):1459-1544. doi: 10.1016 /S0140-6736(16)31012-1.

14. Dwyer-Lindgren L, Stubbs RW, Bertozzi-Villa A, Morozoff C, Callender C, Finegold SB et collab. Variation in life expectancy and mortality by cause among neighbourhoods in King County, WA, USA, 1990-2014: a census tract-level analysis for the Global Burden of Disease Study 2015. Lancet Public Health. 2017;2(9):e400-410. doi: 10.1016/S2468 $-2667(17) 30165-2$.

15. Zhang LR, Rasali D. Life expectancy ranking of Canadians among the populations in selected OECD countries and its disparities among British Columbians. Arch Public Health. 2015;73(1):17. doi: 10.1186/s13690-015 -0065-0.

16. Agence de la santé publique du Canada (ASPC). Outil de données sur les inégalités en santé à l'échelle du Canada, édition 2017. Une initiative conjointe de l'Agence de la santé publique du Canada, du Réseau pancanadien de santé publique, de Statistique Canada et de l'Institut canadien d'information sur la santé [Internet]. Ottawa (Ont.) : ASPC; 2017 [consulté le 5 janvier 2018]. En ligne à : https:// infobase.phac-aspc.gc.ca/health -inequalities/index-fr.aspx

17. Fugelstad A, Ramstedt M, Thiblin I, Johansson LA. Drug-related deaths: Statistics based on death certificates miss one-third of cases. Scand J Public Health. 2017;1-9. doi: 10.1177 /1403494817745187. 